\title{
Guts of giant virus imaged in 3D
}

Reconstruction of mimivirus innards brings X-ray laser images of live cells one step closer.

\section{Davide Castelvecchi}

02 March 2015

What looks like a blurry, misshapen flower is actually the innards of one of the world's largest viruses, imaged in three dimensions using powerful $X$-rays. The same technique could one day create three-dimensional (3D) snapshots of individual molecules, and perhaps even of live bacteria.

The mimivirus (Acanthamoeba polyphaga mimivirus; shown rotating above) carries DNA inside an icosahedral (20-faced) outer shell, and is nearly as large as a typical bacterium. Researchers used the Linac Coherent Light Source (LCLS) at the SLAC National Accelerator Laboratory in Menlo Park, California, to fire powerful X-rays at a single virus particle and build up the 125-nanometreresolution picture of its internal electron density.

The scans - which are published on 2 March in Physical Review Letters ${ }^{1}$ — are a proof of principle that extremely powerful X-ray beams could one day take pictures of small objects that cannot be crystallized, says Janos Hajdu, a molecular biophysicist at Uppsala University in Sweden.

\section{Images without crystals}

Structural biologists routinely fire beams of X-rays at complex molecules and viruses to decode their shapes. But a single molecule does not scatter sufficient X-rays to allow its shape to be reconstructed. In X-ray crystallography, the problem is solved by arranging many copies of the same object into a crystal and looking at repeating patterns in the scattered light. But some molecules are hard to crystallize. And larger, more-complex objects tend to differ from one specimen to the next - for example, genetic material is not arranged in the same way inside all living cells of the same bacterium strain.

The solution could lie in machines known as free-electron lasers, which produce short, densely-packed pulses of X-rays. Each pulse packs in so many high-energy X-ray photons that the machines can — in theory — produce pictures even of single molecules. The LCLS was the first of a handful of such facilities that now exist around the world. 
The beams are so powerful that they destroy any molecule in their path. But some researchers argue that light scattered from a brief enough pulse of X-rays should carry information about a specimen's shape before the molecule itself is destroyed - a principle dubbed 'diffraction before destruction'.

\section{D pictures}

So far, the X-ray lasers have been tested on microscopic crystals and have produced two-dimensional images of large objects such as cyanobacteria $^{2,3}$. But the ultimate goal is to produce fully 3D structures, as X-ray crystallography does. Hajdu and his collaborators tested the idea at the LCLS on the mimivirus in 2011 , but it then took a tour de force of computation to combine several diffraction patterns from many specimens - which, in contrast to the molecules in a crystal, each faced the beam in a different, random orientation — into the 3D image above.

To produce the images, Tomas Ekeberg, a biophysicist at Uppsala University in Sweden, spent years perfecting an algorithm first devised ${ }^{4}$ by researchers at Cornell University in Ithaca, New York. Although that idea dates back to 2009, says Hajdu, until now "it has never been possible to use it on anything meaningful”. Not much can yet be learned from the 3D picture, says Ekeberg, but the scans confirm the expectation that the mimivirus is less densely packed with genetic material than smaller viruses tend to be.

Michael Rossmann, a structural biologist at Purdue University in West Lafayette, Indiana, whose team produced 3D images of mimivirus in 2005 using an electron microscope ${ }^{5}$, says that the $\mathrm{X}$-ray laser technique has yet to show that it can yield more 3D information about viruses than can existing tools that cost a fraction of the LCLS's price. Although, he adds, "I see no reason why they shouldn't eventually get to the kind of resolution they want."

With powerful free-electron lasers scheduled to open in Europe and Japan, as well future upgrades to the LCLS, researchers hope eventually to produce images with resolutions as good as $0.1 \mathrm{~nm}$, says Hajdu. This would be comparable to the best electronmicroscope images, he says, but in contrast to those, it could be done on live organisms. "There is a difference between surgery and autopsy," he says.

Nature | doi:10.1038/nature.2015.17011

\section{References}

1. Ekeberg, T. et al. Phys. Rev. Lett. 114, 098102 (2015).

2. Hantke, M. F. et al. Nature Photonics 8, 943-949 (2014).

3. van der Schot, G. et al. Nature Commun. http://dx.doi.org/10.1038/ncomms6704 (2015).

4. Loh, N.-T. D. \& Elser, V. Phys. Rev. E 80, 026705 (2009).

5. Xiao, C. et al. J. Mol. Biol. 353, 493-496 (2005).

\section{1 comment}

Guest - 2015-03-04 01:44 PM

Why not use cryo-electron tomography? 